\title{
Electroproduction of Charged Pions in the Quark-Fragmentation Region
}

\author{
G. Drews, W. Gebert, F. Janata, P. Joos, A. Ladage, H. Nagel, ${ }^{(a)}$ H. Preissner, and P. Söding \\ II. Institut für Experimentalphysik der Universität Hamburg, Hamburg, Germany, \\ and Deutsches Elektronen -Synchrotron DESY, Hamburg, Germany
}

and

I. Cohen, R. Erickson, F. Messing, (b) E, Nordberg, R. Siemann, J. Smith-Kintner, and P. Stein Cornell University, Ithaca, New York 14853

and

\author{
A. Sadoff \\ Ithaca College, Ithaca, New York 14850 \\ (Received 11 September 1978)
}

\begin{abstract}
This paper presents results of an experiment on hadron production in deep-inelastic electron scattering. Good agreement with the predictions of the quark-parton model is found. The Fragmentation functions for $u$ and $d$ quarks into pions are determined, and comparison is made with other deep-inelastic processes and with recent quark jet parametrizations.
\end{abstract}

In this Letter we present results from a highstatistics experiment on the charged hadrons produced in deep-inelastic electron-proton scattering in a kinematic region dominated by valence quarks. We use the data to test the quark-parton model, to determine quark-fragmentation functions, and to compare with recent jet-model parametrizations.

In the quark-parton model, at sufficiently high $Q^{2}$ and $W$, the cross section for producing hadrons of type $h$ can be expressed as a summation over all quarks by

$$
\frac{1}{\sigma(x)} \frac{d \sigma^{h}}{d z}(x, z)=\frac{\sum e_{i}^{2} q_{i}(x) D_{i}{ }^{h}(z)}{\sum e_{i}{ }^{2} q_{i}(x)}
$$

where $q_{i}(x)$ is the density of quarks of flavor $i$ and charge $e_{i}$ in the target proton. ${ }^{1,2}$ The quarkfragmentation function $D_{i}{ }^{h}(z)$ is the number of hadrons of type $h$ and momentum fraction $z=p^{\text {lab }} /$ $\nu$ per $d z$, arising from the fragmentation of quark $i$. As usual we define $-Q^{2}$ and $\nu$ as the invariant mass squared and laboratory energy of the virtual photon; $W$ is the invariant mass of the finalstate hadrons, and $x=Q^{2} / 2 M \nu$.

The experiment was performed in an $11.5-\mathrm{GeV}$ electron beam at the Wilson Synchrotron Laboratory. The apparatus, consisting of a streamer chamber with nearly $4 \pi$ acceptance triggered by a scattered electron, is described elsewhere. ${ }^{3}$ Since it was not possible to distinguish among hadrons in this experiment, pion-production cross sections were obtained by subtracting kaons and protons on a statistical basis. The contribu- tion of charged kaons was determined from our measured kaon-fragmentation functions ${ }^{3}$ together with the assumptions $D_{u}{ }^{{ }^{-}} / D_{u}{ }^{K^{+}}=D_{u}{ }^{\pi^{-}} / D_{u}{ }^{\pi^{+}}$and $D_{s}{ }^{K^{+}} \ll D_{s}{ }^{K^{-}}$. This contribution, $(10 \pm 3) \%$, is nearly independent of $z$.

The proton subtraction was made using a parametrization of the structure function for proton electroproduction. This parametrization, which is a function of $p_{\perp}, p_{\|}{ }^{*} / p_{\max }{ }^{*}$, and $W$, describes available data ${ }^{4}$ to within $20 \%$. The $p_{\perp}$ dependence was obtained from experiments in a lower $W$ range than our experiment, and the $W$ and $p_{\|} * /$ $p_{\text {max }}{ }^{*}$ dependences came from measurements at $p_{\perp}<0.14 \mathrm{GeV} / c$. With the restriction of $W^{2}>12$ $\mathrm{GeV}^{2}$ for positive hadrons, the proton contribution (before subtraction) is only $(20 \pm 6) \%$ of all charged hadrons at $z=0.3$, and it decreases as $z$ approaches 1 .

Elastic $\rho^{0}$ and $\omega$ production events were identified by kinematic reconstruction and excluded from the data sample. This resulted in a $z$-dependent correction of up to $20 \%$.

Additional corrections of $8 \%$ were made for inefficiencies due to overlapping tracks and a small insensitive region in the streamer chamber, as well as for hadrons misidentified as a bremsstrahlung or Mфller-scattered electrons. Radiation corrections ${ }^{5}$ adjusted $z$ upwards by lowering the value of $\nu$ by $6 \%$ on the average.

To test the quark-parton model we restrict the $x$ range to $x>0.1$, where valence quarks dominate. When we use charge conjugation and isospin invariance and neglect strange quarks from the nucleon's sea, Eq. (1) for the sum of $\pi^{+}$and 
$\pi^{-}$becomes independent of $x$ :

$$
\begin{aligned}
\frac{1}{\sigma(x)}\left(\frac{d \sigma^{\pi^{+}}}{d z}(x, z)+\right. & \left.\frac{d \sigma^{\pi^{-}}}{d z}(x, z)\right) \\
& \cong D_{u}{ }^{\pi^{+}}(z)+D_{u}{ }^{\pi^{-}}(z) .
\end{aligned}
$$

In Fig. 1 we present the left-hand side of Eq. (2) for three regions of $x$, divided by the average of (2) over the full $x$ range at each fixed $z$ value. We conclude that the predicted $x$ independence holds within reasonable accuracy. The limitation of this test is the systematic error associated with the kaon and proton subtractions. Similar previous tests have suffered from either kaon and proton contamination, significant sea-quark contributions, limited $x$ range, or limited $p_{\perp}$ range. ${ }^{6}$

A test of the quark-parton model which is independent of hadron identification uncertainties can be made using only negative hadrons. Restricting the data to $x>0.2$, one can neglect quarks in the nucleon sea and obtain

$$
\frac{1}{\sigma(x)} \frac{d \sigma^{h^{-}}(x, z)}{d z}=\frac{4 q_{u}(x)\left[D_{u}{ }^{\pi^{-}}(z)+D_{u}{ }^{K^{-}}(z)\right]+q_{d}(x)\left[D_{d}{ }^{\pi^{-}}(z)+D_{d}{ }^{K^{-}}(z)\right]}{4 q_{u}(x)+q_{d}(x)}
$$

Since $u$ quarks account for $\sim \frac{8}{9}$ of the cross section nearly independently of $x$, the variation of Eq. (3) with $x$ is small as long as $D_{d}{ }^{{ }^{-}} / D_{u}{ }^{{ }^{-}} \leqslant 4$. If we assume this bound, which is consistent with our data (see below), the net variation of Eq. (3) is less than $10 \%$ for $0.2<x<0.6$. Figure 2 shows this prediction to be well supported by the data.

In Fig. 3 we present our charged-pion data in comparison with corresponding data for $\pi^{0}$ electroproduction ${ }^{7}$ and for $\pi^{ \pm}$production by $e^{+} e^{-}$annihilation $^{8}$ (where $z=E_{h}^{\text {lab }} / E_{\text {beam }}$ ) below the charm threshold, multiplied by 2 and 0.5 , respectively. In the quark-parton model these pion distributions are expected to be equal, apart from a difference of at most $10 \%$ between the $e p$ and the $e^{+} e^{-}$ data due to different contributions from strange quarks. The agreement between the two electroproduction experiments is excellent. The $e^{+} e^{-}$ data agree with the ep data within $\sim 30 \%$, but a somewhat different $z$ dependence is suggested. Comparison of our data with the parametrizations by Sehgal ${ }^{2}$ and by Andersson, Gustafson, and Peterson, ${ }^{9}$ and with the "standard jet model" of Field and Feynman ${ }^{10}$ for fragmenting $u$ or $d$ quarks is also shown in Fig. 3.

To determine the fragmentation functions $D_{u}{ }^{\pi^{ \pm}}$ $(z)$, we have fitted Eq. (1) to our $\pi^{ \pm}$data in bins of $x$ and $z$. The kinematic region was $2<Q^{2}<6$ $\mathrm{GeV}^{2}\left(\left\langle Q^{2}\right\rangle=2.8 \mathrm{GeV}^{2}\right), 0.1<x<0.45(\langle x\rangle=0.2), 12$

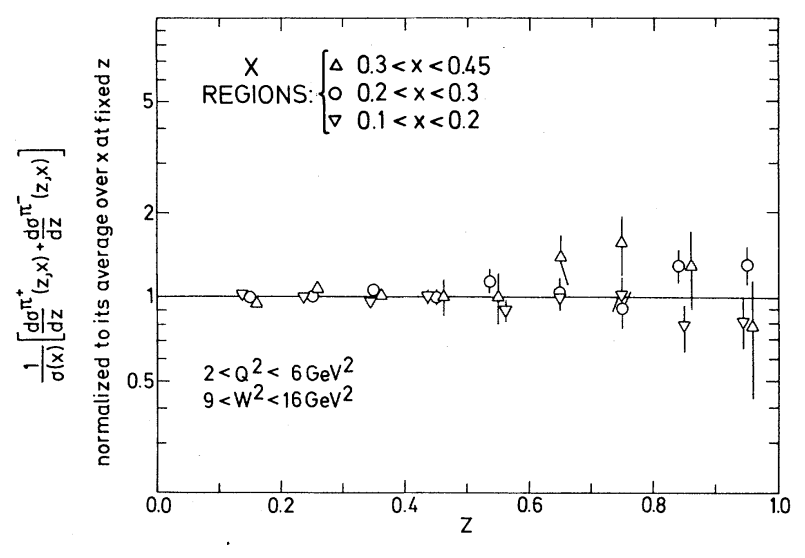

FIG. 1. Sum of the $\pi^{+}$and $\pi^{-}$distributions for different regions of $x$, testing dependence on $x$. The $z$ dependence has been divided out.
$<W^{2}<16 \mathrm{GeV}^{2}$ for $\pi^{+}$, and $9<W^{2}<16 \mathrm{GeV}^{2}$ for $\pi^{-}$. The quark density functions $q_{i}(x)$ were taken from Field and Feynman ${ }^{11}$; the sea-quark parametrization is not critical in our $x$ range. The fit gives

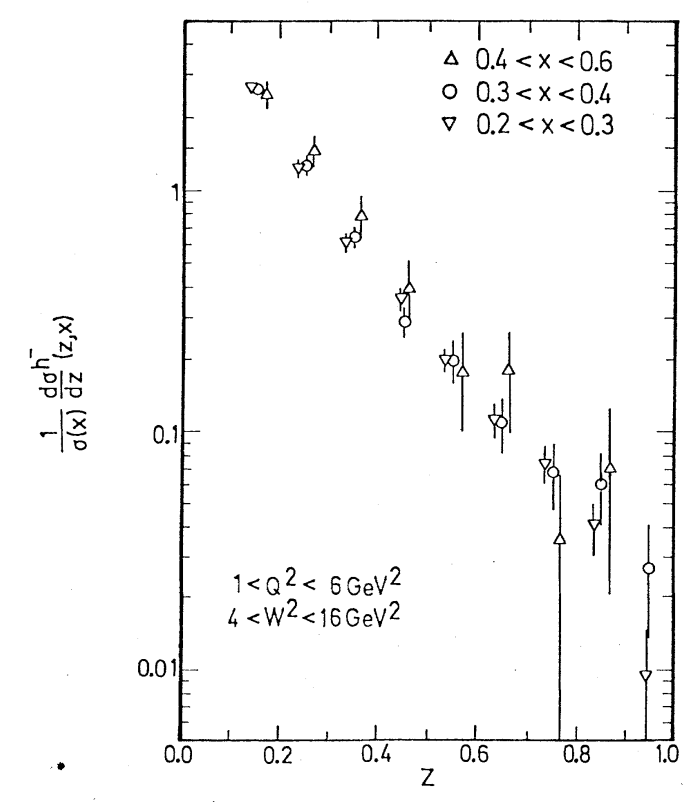

FIG. 2. The negative-hadron distribution for different regions of $x$. 


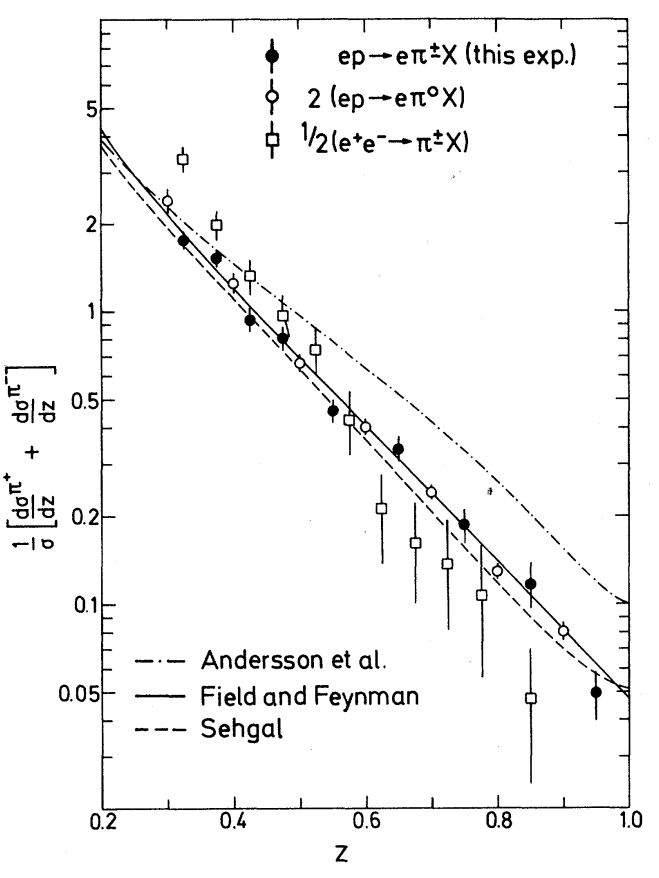

FIG. 3. Sum of the $\pi^{+}$and $\pi^{-}$distributions for this experiment, compared with measurements from other deep-inelastic processes (Refs. 7 and 8) and with various quark-parton model parametrizations (Refs. 2, 9, and 10). The kinematic range of this experiment is 2 $<Q^{2}<6 \mathrm{GeV}^{2}$; $12<W^{2}<16 \mathrm{GeV}^{2}$ for $\pi^{+}, 9<W^{2}<16 \mathrm{GeV}^{2}$ for $\pi^{-}$; and $0.1<x<0.45$ 。

$\chi^{2}=108$ for 90 degrees of freedom, confirming that Eq. (1) of the quark-parton model is consistent with our data.

The fitted fragmentation functions are shown in Fig. 4. They are in agreement with those proposed by Field and Feynman. ${ }^{10}$ In particular, their predicted limiting behavior at $z \rightarrow 1$ is consistent with our results. For comparison, results from the reactions $\bar{\nu} p \rightarrow \mu^{+} h^{-} X$ and $\nu p \rightarrow \mu^{-} h^{-} X$ are also shown. ${ }^{12,13}$ These reactions are expected to be dominated by $D_{u}{ }^{\pi^{+}}$and $D_{u}{ }^{\pi^{-}}$, respectively, but as a result of sea-quark contributions and $K^{-}$production, the agreement with our data is not expected to be perfect.

After we have determined $D_{u}{ }^{\pi^{ \pm}}(z), D_{u}{ }^{\pi^{0}}(z)$ can be calculated. The momentum sum rule for forward-going pions gives

$$
\sum_{\pi^{+}, \pi^{-}, \pi^{0}} \int_{0.3}^{1} z D_{u}{ }^{\pi}(z) d z=0.25 \pm 0.01
$$

Using our fragmentation functions for kaons, ${ }^{3}$ we obtain

$$
\sum_{K^{+}, K^{-}, K^{0}, \bar{K}^{0}} \int_{0.3}^{1} z D_{u}{ }^{K}(z) d z=0.028 \pm 0.006
$$

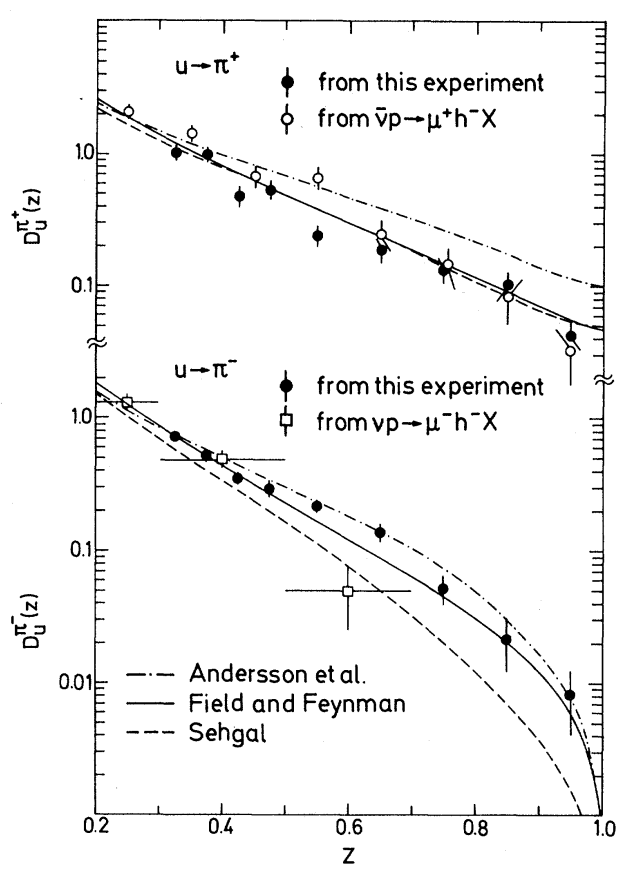

FIG. 4. Fragmentation functions of $u$ quarks into $\pi^{+}$ and $\pi^{-}$compared with results from $\nu p$ and $\overline{\nu p}$ experiments (Refs. 12 and 13), and with the parametrizations by Sehgal, by Andersson, Gustafsson, and Peterson, and by Field and Feynman (Refs. 2, 9, and 10)。

Adding Eqs. (4) and (5) shows that pions and kaons with $z>0.3$ carry about $30 \%$ of the total momentum of the parent quark.

In Fig. 5, the $z$ distributions of the fastest and second-fastest charged hadrons (including $K$ 's

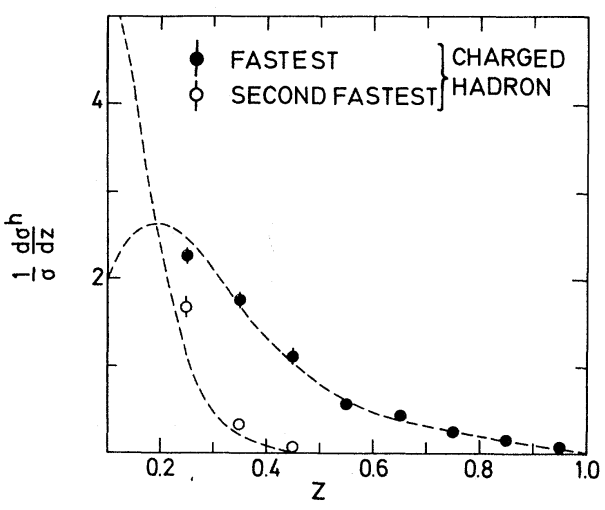

FIG. 5. $z$ distribution of the fastest and second-fastest hadrons for the kinematic region $2<Q^{2}<6 \mathrm{GeV}^{2}, 12$ $<W^{2}<16 \mathrm{GeV}^{2}$, and $0.1<x<0.45$, where $u$ quarks are expected to dominate, compared with the prediction by Field and Feynman (Ref. 10) for $u$-quark jets of $10-\mathrm{GeV}$ total momentum. 
but not protons) are shown. Comparison with the prediction for "standard jets" by Field and Feyn$\operatorname{man}^{10}$ again shows rather good agreement. In the region $z>0.3$, the fastest and second-fastest charged hadrons carry $(21 \pm 1) \%$ and $(1.7 \pm 0.2) \%$ of the total momentum.

In summary, our investigation of electroproduced final states has shown good agreement with the quark-parton model. Comparison with other deep-inelastic processes suggests a common mechanism for hadron production. In addition, we find our data in agreement with the "standard jet" parametrization of Field and Feynman.

This work was supported in part by the Bundesministerium für Forschung und Technologie and the National Science Foundation. We gratefully acknowledge the support of Professor B. D. McDaniel, Professor H. Schopper, Professor H. Spitzer, and Professor G. Weber, and the staffs of DESY and the Wilson Synchrotron Laboratory.

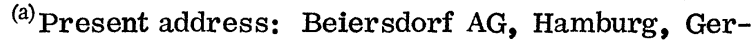
many.

(b) Present address: Carnegie-Mellon University, Pittsburgh, Penn. 15213.

${ }^{1}$ R. P. Feynman, Photon-Hadron Interactions (W. A. Benjamin, Reading, Mass., 1972).

${ }^{2}$ L. M. Sehgal, in Proceedings of the 1977 Intermation-
}

al Symposium on Lepton and Photon Interactions at High Energies, edited by F. Gutbrod (Deutsches Elektronen Synchrotron, Hamburg, Germany, 1977).

${ }^{3} \mathrm{I}$ 。 Cohen et al., Phys. Rev. Lett。40, 1614 (1978).

${ }^{4} \mathrm{H}$. Ackermann et al., Nucl. Phys. B120, 365 (1977); J. C. Alder et al., Nucl. Phys. B46, 415 (1972); C. J. Bebek et al., Phys. Rev. Lett. 32, 27 (1974); C. J. Bebek et al., Phys. Rev. D 15, 3077 (1977); A. Browman et al., Phys. Rev. Lett. 37, 651 (1976).

${ }^{5}$ L. Mo and P. Tsai, Rev. Mod. Phys. 41, 205 (1969).

${ }^{6}$ C. del Papa et al., Phys。Rev. D 15, 2425 (1977);

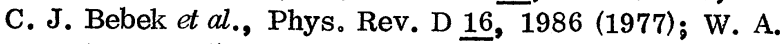
Loomis et al., "Hadron Production in Muon-Proton and Muon-Deuterium Collisions" (to be published); J. F. Martin et al., Phys. Rev. Lett. 38, 1193 (1977); J. M. Scarr et al., DESY Report No。 DESY 77/77, 1977 (unpublished).

${ }^{7} \mathrm{C}$. Berger et al., Phys. Lett. $\underline{70 \mathrm{~B}}, 47$ (1977), and private communication.

${ }^{8}$ R. Brandelik et al., Phys. Lett. 67B, 358 (1977), and private communication.

${ }^{9}$ B. Andersson, G. Gustafson, and C. Peterson, Nucl. Phys. B135, 273 (1978).

${ }_{10}$ R. D。 Field and R。 P. Feynman, California Institute of Technology Report No. CALT-68-618, 1977 (to be published).

${ }^{11}$ R. D. Field and R. P. Feynman, Phys. Rev. D $\underline{15}$, 2590 (1977).

${ }^{12}$ M. Derrick et al., Argonne National Laboratory Report No. ANL-HEP-PR-77-39, 1977 (to be published).

${ }^{13} \mathrm{~J}$. C. Vander Velde, in Proceedings of the XII Rencontre de Moriond, 1977, edited by J. Tran Thanh Van (Centre National de la Recherche Scientifique, Orsay, France, 1977).

\title{
Energy Dependence of the Spin-Spin Correlation Parameter $C_{L L}=(L, L ; 0,0)$ in $p$ - $p$ Elastic Scattering around $\theta_{\text {c.m. }}=\mathbf{9 0 ^ { \circ }}$
}

I. P. Auer, A. Beretvas, ${ }^{(a)}$ E. Colton, H. Halpern, ${ }^{(b)}$ D. Hill, K. Nield, B. Sandler, ${ }^{(c)}$ H. Spinka, G. Theodosiou, D. Underwood, Y. Watanabe, ${ }^{(d)}$ and A. Yokosawa Argonne National Laboratory, Argonne, Illinois 60439

(Received 15 August 1978)

\begin{abstract}
We have measured the spin-spin correlation parameter $C_{L L}=(L, L ; 0,0)$ in $p-p$ elastic scattering around $\theta_{\mathrm{c}, \mathrm{m}_{.}}=90^{\circ}$ from $p_{1 \mathrm{ab}}=1.0$ to $3.0 \mathrm{GeV} / c$. We observe a rapid energy dependence in $C_{L L}$ and describe our interpretation of the results.
\end{abstract}

We have previously reported measurements of the total-cross-section difference, $\Delta \sigma_{L}$, for proton-proton scattering using a beam and a target that were both longitudinally polarized., $2 \mathrm{~A}$ striking energy dependence observed in $\Delta \sigma_{L}$ was interpreted as evidence for the formation of diproton resonances. ${ }^{3}$ During the course of these measurements, we simultaneously measured the spin-spin correlation parameter $C_{L L}=(L, L ; 0,0)$ in $p-p$ elastic scattering for $70^{\circ} \leqslant \theta_{c_{0} \mathrm{~m}_{0}} \leqslant 110^{\circ}$ at $p_{\text {lab }}=1.0-3.0 \mathrm{GeV} / c .{ }^{4}$ The experimental layout is shown in Fig. 1. Besides the longitudinally polarized beam and target described in earlier references, the apparatus consisted of a set of triggering scintillation counters, and an array of multiwire proportional chambers which were used to reconstruct the incident- and outgoing-proton trajectories. The detectors were positioned so that they would not interfere with $\Delta \sigma_{L}$ measurements, thus covering $\theta_{\text {c. } \mathrm{m}_{\mathrm{o}}}$ near $90^{\circ}$. 